\title{
La novela-diario como estructura simbólica en Tlactocatzine, del jardín de Flandes, de Carlos Fuentes.
}

\section{The Diary Novel as Symbolic Structure in Tlactocatzine, del jardin de Flandes by Carlos Fuentes.}

Esta obra está bajo una Licencia Creative Commons Atribución 4.0 Internacional. DOI: $10.32870 /$ sincronia.axxiii.n75.10a19

\section{Joo Kyeong Kim}

Universidad Nacional de Seúl

anita92@snu.ac.kr

(COREA DEL SUR)

Recibido: $10 / 09 / 2018$

Revisado: $22 / 11 / 2018$

Aprobado: $10 / 12 / 2018$

\section{RESUMEN}

El presente estudio se enfoca en la estructura de novela-diario de "Tlactocatzine, del jardín de Flandes", uno de los primeros cuentos publicados por Carlos Fuentes, en 1954. Entre otras características, la novela-diario requiere de un narrador en primera persona y narraciones fragmentarias que pueden ir fechadas. En este artículo, analizaremos el texto "Tlactocatzine", considerando los presupuestos de la novela-diario, ya que contiene ambos elementos: un narradorprotagonista y narraciones fragmentarias junto con fechas específicas. Revisaremos cuál sería el objetivo de configurar la narración con esta estructura, en dos apartados principales. Demostraremos que el primer propósito es la asimilación del lector con el protagonista, ya que dicha estructura composicional es un mecanismo efectivo para acentuar los sentimientos y provocar la ambigüedad. El segundo propósito es mostrar que las fechas funcionan simbólicamente indicando el equinoccio y el periodo correspondiente de la fiesta azteca. El equinoccio funciona como punto de 
bifurcación no solo de las estaciones, sino también de los estados psicológicos del protagonista, y los días de las entradas del diario corresponden a las fiestas del regreso de los dioses. Concluimos que Carlos Fuentes utilizó esta configuración estructural con el propósito de reducir hasta eliminar la distancia en varias maneras.

Palabras clave: Novela-diario. Narrador. Género gótico. Equinoccio. Reencarnación.

\section{ABSTRACT}

The present study has its focus on the diary novel structure of "Tlactocatzine, del jardín de Flandes", which is one of Carlos Fuentes' first short novels published in 1954. For a novel to be a "diary novel", it should have first-person narrator and fragmented narration, possibly with dates. Here, we will analyze "Tlactocatzine" as a diary novel, as this story has both first-person protagonist-narrator, and fragmented narration with specific dates. We will investigate the objectives for which our writer used this rarely used structure in his narration. It is analyzed largely by two points. On one hand, Fuentes wanted the readers to identify themselves with the story's main character. This compositional structure is a very effective tool to foment emotions and to provoke ambiguity. In addition, the specified dates indicate the equinox and the Aztec fiesta period. The equinox is a point of bifurcation, not only of the season, but also of the protagonist's psychological state. Also, the dates of the diary entries correspond to the fiesta celebrating the return of the Aztec gods. As such, Fuentes utilized this special structure for a purpose, which is to reduce, almost to eliminate the gap in many respects.

Keywords: Diary novel. Narrator. Gothic genre. Equinox. Reencarnation.

\section{Introducción}

El narrador es "el concepto fundamental" de todo texto narrativo (Bal, 1990, p. 126) y "el elemento central del relato" (Garrido Domínguez, 1996, p. 105). Garrido Domínguez (1996, p. 110) sostiene que el narrador representa un papel crucial en el texto narrativo, así que sería imposible escribir un relato sin narrador. Puede hallarse dentro o fuera del discurso, puede actuar o simplemente percibir (Bal, 1990, p. 131). Uno de sus papeles más importantes es la entrega de información a los lectores. Garrido 
Domínguez cita a Todorov para afirmar que "es quien disimula o revela los pensamientos de los personajes, haciéndonos participar así de su concepción de la psicología” (1990, p. 111).

Carlos Fuentes experimentó con varios tipos de narradores desde sus primeras obras. En Aura, usa de manera muy particular la segunda persona: "Unlike conventional omniscient narrators, who address their readers either directly or indirectly, this Narrator addresses the protagonist Felipe familiarly, using the second person pronoun tú." (Rojas, 1978, p. 859). Desde el inicio de la novela se percibe dicha forma de narrar: "Lees ese anuncio: una oferta de esa naturaleza no se hace todos los días. Lees y relees el aviso. Parece dirigido a ti, a nadie más" (Fuentes, 2007b, p. 131). ${ }^{1}$

En el caso de La muerte de Artemio Cruz, utiliza tres tipos de narradores, en primera, segunda y tercera persona "para conjugar una visión completa -interior y exterior- de un personaje que [...] es la síntesis de distintas épocas y sectores sociales de México" (Tello Hernández, 2008, p. 89). La novela empieza con narrador en primera persona: "Yo despierto... Me despierta el contacto de ese objeto frío con el miembro. [...] Permanezco con los ojos cerrados" (Fuentes, 1970, p. 9). Pero, en el siguiente capítulo, el narrador está en segunda persona: "Tú te sentirás satisfecho de imponerte a ellos; confiésalo: te impusiste para que te admitieran como su par" (Fuentes, 1970, p. 32), y en el tercero, aparece el narrador del pasado histórico en tercera persona.

Como agente de texto narrativo, el narrador más la focalización constituyen juntos la narración (Bal, pp. 125-126). De ahí que el método de la narración o, en este caso, el empleo de la forma de diario, determine elementos cruciales en el cuento. Se puede notar el interés del autor por diferentes tipos de narraciones desde su primer volumen de cuentos, Los días enmascarados. Hay pocos relatos de Fuentes con la forma de diario, pero dos de este libro presentan esa estructura. Ciertamente, tiene sus ventajas. No es necesario que los acontecimientos sean continuos. Carlos Fuentes, joven escritor en 1954, pudo haber preferido usar la forma de diario porque es un método de narración aparentemente más sencilla. Sin embargo, es interesante que mostrara diferentes estilos de novela-diario en dos cuentos, "Chac Mool" y "Tlactocatzine, del jardín de Flandes". "Chac Mool" toma la forma de diario, pero diferente de nuestro cuento de estudio. El narrador, un amigo de Filiberto, lee el diario del protagonista como un

\footnotetext{
${ }^{1}$ Citamos por esta edición. Todas cursivas de las citas son nuestras e indicaremos cuando pertenezcan al texto.
} 
lector más. Entre los apuntes, el amigo interviene para dar explicaciones que no son parte del diario de Filiberto: "Hasta aquí, la escritura de Filiberto era la vieja, la que tantas veces vi en memoranda y formas, ancha y ovalada. La entrada del 25 de agosto, parecía escrita por otra persona" (Fuentes, 1982, p. 19). Como los lectores no pueden notar el cambio de la escritura, el amigo-narrador se encarga de explicarlo. Por el contrario, en "Tlactocatzine", el diario del protagonista se cuenta directamente a los lectores. Fuentes aprovecha varios recursos narrativos en sus libros, para conseguir diversos efectos. En este artículo, en primer lugar, veremos las características de la novela-diario. Después, analizaremos los efectos de la configuración narrativa en forma de diario para, finalmente, mostrar las funciones simbólicas de las fechas en "Tlactocatzine, del jardín de Flandes".

\section{La novela-diario}

El debate en torno a la definición de la novela-diario se mantiene vigente. Algunos críticos dicen que la novela-diario constituye un subgénero. Según Prince (1975, p. 477), "constitute a sub-genre, a species, a particular subset of the set of all fiction [...], they are specifically characterized by certain features not found in other types of fiction". Pero otros estudiosos argumentan que es solo una forma narrativa. Abbott (1982, p. 20) afirma: "My framing argument is that diary fiction is best conceived not as a genre but as fiction employing a particular narrative device". En su opinión, la novela-diario es una estrategia que pueden adoptar los autores. Por otro lado, Beltrán Almería (2011, p. 17) señala: “[...] la novela no es un género ni un subgénero. Pero tampoco un tipo especial de narración [...], el diario aporta a la novela un eslabón en la evolución de sus formas compositivas".

Aunque es difícil de definir una novela-diario, hay ciertas características que se aceptan extensivamente. Primera, es esencial que haya una presencia pretendida e incuestionable del estilo del diario (Field, 1989, p. 5). ${ }^{2}$ Además, habrá un narrador-protagonista: "The diary novel is a roman personnel, an Ich-Romank, a first-person novel in which the narrator is a protagonist in the events he

\footnotetext{
${ }^{2} C f$. "[...] one should be expecting a substantial or indeed overwhelming presence in the novel of the purported diary." (Field, 1989, p. 5).
} 
records" (Prince, 1975, p. 477). Finalmente, las narraciones serán fragmentarias y un apunte describirá los acontecimientos pasados, como afirma Prince.

Según las características mencionadas, "Tlactocatzine, del jardín de Flandes", tercer cuento de Los días enmascarados, seguiría el estilo de una novela-diario. "Tlactocatzine" es en sí mismo un diario del protagonista. El diario empieza el 19 de septiembre y continúa por seis días, hasta el 24 del mismo mes. En el primer día, el protagonista se traslada a una mansión. Unos días después, encuentra a una anciana en el jardín de la biblioteca. El último día, el narrador termina secuestrado en la mansión y la mujer queda satisfecha por haber cumplido su objetivo.

La narración transcurre en primera persona, explicando y escribiendo en el diario los acontecimientos, además de la emoción y los pensamientos del protagonista: "Esto, como digo, creía yo entonces. No fue poca mi sorpresa cuando el licenciado me comunicó sus intenciones" (Fuentes, 1982, p. 34). ${ }^{3}$ En la cita anterior, se destaca la reacción del narrador-personaje directamente, como si contara su historia a un lector hipotético. También, en este cuento, el narrador describe lo que pasó a partir de la entrada precedente del mismo diario, coincidiendo con otra de las características de la novela-diario: "23 Sept. Sonaban las seis cuando escuché música en el salón [...], ella estaba en el jardín [...]. 24 Sept. Después de la aparición del atardecer, recobré el conocimiento sentado en el sillón de la biblioteca" (pp. 42-43). El texto del día 23 explica lo ocurrido hasta el encuentro entre el protagonista y la mujer misteriosa. El siguiente fragmento cuenta qué hizo el narrador-personaje después del encuentro. "Tlactocatzine" está configurado estructuralmente como una forma especial de novela-diario, revisaremos cuál seria la intención de dicha forma de estructura.

\section{Dos influencias sobre los lectores}

\subsection{Sentimiento y misterio}

Pérez (1997, p. 9) señala que algunos cuentos de Carlos Fuentes, incluyendo "Tlactocatzine, del jardín de Flandes", "ejemplifican la evolución posterior de la tradición gótica, incorporando o modificando elementos típicos del género". La literatura inglesa, incluyendo la gótica tuvo mucha influencia sobre

\footnotetext{
${ }^{3}$ Citamos por esta edición. En adelante, se indicarán entre paréntesis las páginas de las citas.
} 
Fuentes (Pérez, 1997, p. 10). Por su parte, Gutiérrez-Mouat (1985, p. 47) sostiene que el autor asumió dicho género gótico "de manera consciente y crítica". En los relatos del escritor mexicano, los papeles de los sexos se invierten. Según Pérez (1997, p. 10), las ficciones góticas tradicionales prefieren "una heroína joven, bella, inocente, cándida, vulnerable". En cambio, Fuentes se apropia de este carácter femenino para sus protagonistas masculinos (Pérez, 1997, p. 12). En "Tlactocatzine", el joven protagonista se traslada a la vieja mansión y es la mujer la que toma la iniciativa de acciones que provocan temor o suspenso, mientras que el hombre es la víctima al permanecer encerrado en la mansión y bajo el control femenino.

Entre las características del género gótico está el que los lectores compartan los sentimientos del protagonista. Muñoz-Basols (2003, p. 73) subraya que se trata de "una «literatura de sensaciones» que, al adentrar al lector en la dimensión psicológica de los protagonistas, incorpora en el entramado narrativo un plano descriptivo más cercano al lector de lo habitual". Y agrega que "el uso de la primera persona permite una identificación fácil entre el lector y el personaje ya que el yo pertenece a todos" (Muñoz-Basols, 2003, p. 73). Pérez (1997, p. 13) afirma, igualmente, que la literatura gótica suele emplear una "voz narradora de primera persona que comunica directamente sus reacciones de duda, vacilación, y sensación de peligro o temor". Por medio de dicha estructura, los lectores pueden conocer los sentimientos y los pensamientos del protagonista. Según el análisis de Abbott $(1982$, p. 20) sobre la novela-diario: "The first is the effect of immediacy: that is, the illusion of being there, of no gap in time between the event and the rendering of it".

En ese sentido, se justificaría el uso del diario en "Tlactocatzine". El lector puede saber qué sintió el protagonista por las referencias sensoriales que se incluyen cuando describe de una manera directa y minuciosa sus reacciones al ver el jardín por primera vez, por ejemplo:

[...] de pie en el centro del jardín, cerré los ojos [...], tabaco javanés y aceras mojadas [...]. iMemling, por una de sus ventanas había yo visto este mismo paisaje, entre las pupilas de una virgen y el reflejo de los cobres! [...] ¡El jardín no estaba en México! (pp. 38-39) 
Más adelante, el narrador-personaje explica con igual detalle qué hizo y cómo se sintió en su primer encuentro con la anciana:

Luego, sentí el ruido sordo, el zumbido que parecía salir de sí mismo, y levanté la cara. En el jardín, casi frente a la mía, otra cara, levemente ladeada, observaba mis ojos. Un resorte instintivo me hizo saltar hacia atrás [...], escondí entre los dedos mis ojos. (pp. 41-42)

Esta relación directa entre el protagonista y el lector facilita la empatía entre ambas instancias. Pérez (1997, p. 13) cita a Freud para describir este rasgo de compartir el "desconocimiento y misterio, temor a lo desconocido, lo anormal", como lo "uncanny". Podríamos afirmar, entonces, que "Tlactocatzine" emplea la estructura de la novela-diario donde el narrador está en primera persona relatando la historia en fragmentos, sumada a la característica de la literatura gótica que utiliza un punto de vista tal que los lectores sienten y comparten lo misterioso.

\subsection{La ambigüedad}

En los cuentos con narrador-personaje como "Tlactocatzine", la verdad que se cuenta es solamente la de dicho personaje. No existe otra versión más que la suya porque solo enuncia los hechos, desde su punto de vista. En este tipo de cuentos, escuchamos una versión única de la historia. Desde el punto de vista de otro personaje, la historia podría ser diferente:

Algunas novelas dramatizan esta falta de objetividad en la figura de un narrador enfáticamente desconfiable que selecciona los hechos que va a relatar de acuerdo a un proyecto personal como en el caso del cronista anónimo de Terra Nostra de Carlos Fuentes, o de Cristóbal Colón en El arpa y la sombra. (Quintana Millamoto, 2008, p. 34) 
La "falta de objetividad" se acentúa en las novelas-diario. Abbott señala que una de las grandes ventajas de la forma del diario es el confinamiento del lector en el mundo interno de un ego y, además, el recurso para excluir la perspectiva del otro: "In diary fiction, one is encouraged by the form itself to let go of the perspective of the other." (Abbott, 1982, p. 17). Este sería el caso de "Tlactocatzine". Casi no hay información sobre el narrador. No sabemos su nombre, ni su edad, ni su caracterización. Además, el texto comprende solamente lo que el narrador-personaje había escrito, por eso el punto de vista carece de objetividad.

Otro elemento ambiguo en el cuento es el final abierto, ya que no sabemos qué ocurre con el protagonista. Esta ambigüedad en el final es posible gracias a la estructura del diario, como sugiere Abbott (1982, p. 23):

The retrospective narrator may also, of course, be dead, but if his death is caused in any way by the events he narrates, it has been sufficiently delayed to allow him to record the whole story [...]. But in diaries, the hand, now living [...], may at any moment be stilled.

Brushwood aplica esta idea a Los días enmascarados. Cuatro de los seis cuentos de la colección cuentan con un narrador en primera persona. El crítico enfatiza que los narradores, aunque todos sean en primera persona, tienen varias funciones y adelantan finales diferentes (Brushwood, 1982, p. 19). En el caso de "Chac Mool", el narrador-personaje refiere la muerte de Filiberto. Aunque presenta la forma de diario y un narrador en primera persona, como "Tlactocatzine", el destino de Filiberto se conoce de antemano. En cambio, "Tlactocatzine, del jardín de Flandes" termina con la voz de la mujer y con la inscripción sobre el escudo revelando que se trata de Carlota, la emperatriz de México durante la Intervención Francesa, en el siglo XIX. No hay información en el diario sobre qué sucede después de la confinación del protagonista en el último día del diario.

\section{Significado de las fechas}


Según la discusión sobre la novela-diario, las fechas no son una condición esencial. Field (1989, p. 1) propone que no es necesario que aparezcan todas las fechas. La estructura de la novela-diario no depende de la cronología y Field recurre a los prototipos de la literatura francesa para sostener su afirmación. El cuento "Chac Mool" no tiene fechas. Cada fragmento está separado por un espacio entre líneas. Presumimos la temporada y el transcurso del tiempo en este cuento por palabras como "Semana Santa", "sábado de Gloria" (p. 9), o "las Iluvias excepcionalmente fuertes de ese verano" (p. 19). Cabría preguntarnos, ¿̇por qué hay fechas, ¿cuál es su función en "Tlactocatzine, del jardín de Flandes”?

\subsection{La función del equinoccio}

La mitad del diario, aproximadamente, es el 21 de septiembre, fecha que coincide con el equinoccio. Durante los equinoccios, "los días son iguales a las noches" (Ledesma Jimeno, 2011, p. 43). El de septiembre señala el cambio de las estaciones, de verano a otoño. Por las fechas, podemos saber exactamente cuándo es el día de equinoccio porque el narrador-protagonista lo señala: "20 Sept. [...] Mañana es el equinoccio" (p. 37). Aunque hay dos o más apuntes cada día, pero son fácilmente identificables las entradas correspondientes al equinoccio porque todas están fechadas.

En la tradición de los antiguos mexicanos, el equinoccio tenía especial importancia (Pérez, 1997, p. 17). Las civilizaciones mesoamericanas poseían un vasto conocimiento de la astronomía. Los movimientos del sol tenían mucho significado, y tanto los solsticios como los equinoccios eran días especiales en su calendario. Kitchel (n.d.) destaca que los mayas realizaban homenajes a los solsticios y a los equinoccios. En el mismo sentido, Tomasini (2007, p. 81) afirma:

[...] algunos de sus principales monumentos se encuentran orientados de tal manera que permiten [...] predecir exactamente el momento de los solsticios y de los equinoccios [...], los fenómenos celestes eran considerados como manifestaciones de la voluntad divina.

Pérez (1997, p. 17) señala que todos los relatos de Los días enmascarados "exhiben rasgos fantásticos, personajes en transformación, que se relacionan de alguna manera con el calendario". En esos términos, 
el equinoccio en "Tlactocatzine" significaría cambios relacionados con la transición de las estaciones, del verano al otoño. El 20 de septiembre, día anterior al equinoccio, el narrador-personaje nota la llegada del otoño: "algunas hojas han caído del emparrado [...], otras, comienzan a dorarse, y la lluvia incesante parece lavar lo verde" (p. 37). Mientras que el tiempo, las hojas, el ambiente del jardín indican el cambio de la estación, el protagonista sufre también un cambio. El lector puede notar que el destino del narrador-personaje cambia a partir del equinoccio. Según Liebowitz Knapp (1986, pp. 132-133), Ilega al punto de la conversión: "He mentions in his diary the advent of the equinox [...]. So, too, has he [...], reached the midpoint". El día 19, el protagonista no encuentra las llaves del ventanal que mira al jardín y debe permanecer dentro de la casa. Sin embargo, logra salir en el equinoccio:

21 Sept. Por fin he logrado abrir la ventana de la biblioteca. Salí al jardín. Sigue esta llovizna, imperceptible y pertinaz [...], las siemprevivas no son las que conozco [...]. En el jardín, casi frente a la mía, otra cara, levemente ladeada, observaba mis ojos. (pp. 38-39)

Justamente ese día, 21 de septiembre, encuentra a una persona misteriosa en el jardín. Y paso a paso, entra al mundo del pasado, de la irrealidad y de la muerte. Pérez $(1997$, p. 18) concluye su análisis comparando al narrador-protagonista del cuento con "las típicas víctimas del vampiro clásico, ni vivas ni muertas sino condenadas a existir en otro mundo infernal". No obstante, podemos observar en nuestro análisis que, con el cambio de su destino, el protagonista empieza a perder la razón. Se puede deducir que posee un alto nivel de cultura, ya que conoce bien a los pintores europeos y asocia las cadencias de Rodenbach con el jardín, el primer día que se traslada. Sin embargo, después del equinoccio, "el jardín hipnotiza al actor, quien carece de voluntad para resistir el hechizo" (García Gutiérrez Vélez, 1978, p. 46), y comienza a actuar de una manera inexplicable. Al día siguiente, parece capaz de todavía hacer preguntas lógicas, aunque ya no actúa de manera razonable: 
22 Sept. ... ipero si estoy viviendo en mi ciudad, entre mi gente! ¿por qué no puedo arrancarme de esta casa, diría mejor, de mi puesto en la ventana que mira al jardín? ${ }^{4}$ 22 Sept. No me voy a asustar porque alguien saltó la tapia y entró al jardín. [...] Era una viejecita... tendría ochenta años, cuando menos, ¿pero ¿cómo se atrevía a entrar, o por dónde entraba? (p. 40)

Después, comienza a esperarla, "23 Sept. Debe venir, como ayer y anteayer, a la caída del sol. Hoy le dirigirlé la palabra." (p. 42), no se extraña de que la música esté tocando automáticamente y, siguiendo un impulso, pasa al jardín cuando encuentra a la anciana: "23 Sept. [...] Sonaban las seis cuando escuché música en el salón; era el famoso Pleyel, tocando valses. A medida que me acerqué, el ruido cesó. Regresé a la biblioteca: ella estaba en el jardín [...]. Abrí la ventana; salí" (p. 42). En vez de escapar, el protagonista espera una señal de la anciana y, cuando la ve, sale a su encuentro en el jardín. Podemos concluir que, desde la fecha del equinoccio, el protagonista sufre una transformación en su manera de actuar al punto que parecería enajenado o que ha perdido la razón.

\subsection{La reencarnación}

Otra función de las fechas tendría que ver con la historia mexicana. Las fechas infieren que el protagonista es o va a ser la reencarnación de Maximiliano I o de un rey azteca. La anciana que hechiza al narrador es la emperatriz Carlota y piensa que el huésped de la mansión es su esposo, Maximiliano de Habsburgo. Maximiliano fue emperador de México por poco tiempo, de 1864 a 1867, durante la intervención francesa. No sabemos exactamente qué sucede después del final del cuento, pero se puede deducir que el protagonista asume el papel del Emperador mediante reencarnación. Ciertamente, el protagonista ya es o, al menos, va a transformarse en Maximiliano. 'Tlactocatzine', el nombre que encuentra en la carta antigua deslizada en el piso y que usa la Emperatriz, está asociado con dos personas. En la historia de México se registra que este nombre lo usaron los indios para recibir

\footnotetext{
${ }^{4}$ Las minúsculas de ambas frases entre signos de exclamación e interrogación pertenecen al texto y se mantienen en la edición de Cuentos sobrenaturales (Fuentes, 2007a, p. 44), donde se incluye también este mismo cuento.
} 
al nuevo emperador. Cuando llegaron a uno de pueblos en su viaje de Veracruz -puerto de desembarcohacia la Ciudad de México, el alcalde dio un discurso al emperador en la lengua de los aztecas: "No mahustililoni tlactocatziné [...]. Nis tiquinopielia inin maxochtzintl, quen se machiotl in tetlasotla litzin, mitzmo maquilia mo xocotitlan coneztzitzihua" (Hall, 1868, p. 118). ${ }^{5}$ En náhuatl, "tlactocatzine" significa emperador. Las flores que le entregaron eran 'siemprevivas', típicas del Día de Muertos. Esas mismas flores eran las que olían más fuerte cada día, invadiendo con su aroma la mansión desde el jardín.

En un segundo momento, hay evidencia que podría tratarse de un rey azteca. 'Tlactocatzine' "recuerda el nombre de un rey chichimeca, Tlaltetcatzin, quien «gobernó a los de Tezcuco ochenta días no más»" (Simonson, 2009, pp. 25-26). Además, los eventos del cuento transcurren durante los últimos días del mes de septiembre, correspondientes a "las Fiestas del regreso de los dioses" (Simonson, 2009, p. 25). Asimismo, la duración de la preparación, seis días incluyendo el día de la fiesta, coincide con el período del cuento-diario de seis días. La preparación empieza el 15, y la fiesta es al final del mes, el día 20: "A quince días andados deste mes enramaban unos altares que ellos llamaban momoztli con cañas atadas de tres en tres", según refiere fray Bernardino de Sahagún (2000, p. 236).

\section{Conclusión}

Carlos Fuentes, en Cervantes o la crítica de la lectura (1976), afirmó: "La literatura es la utopía que quisiera reducir [las] separaciones" (citado por López González, 2011, p. 19). Dicha premisa de su poética debemos remontarla a su primer libro, Los días enmascarados. En "Tlactocatzine, del jardín de Flandes", Fuentes trata de empatizar al lector con el protagonista, de reunir vida y muerte, presente y pasado, cultura azteca y europea en un mismo texto. Y la forma del diario es el mecanismo que facilitaría el enlace.

En primer lugar, mediante el narrador en primera persona, se fortalece el vínculo entre lector y protagonista. Simpatizamos con el protagonista, sintiendo lo misterioso desde su experiencia. Esta

\footnotetext{
${ }^{5}$ El texto consultado consigna la traducción en inglés: “Our honorable Emperor [...]. Here is this flower; see in it the sign of our love. Your sons of Naranjal give it to you" (Hall, 1868, pp. 118-119).
} 
conjunción continúa hasta el final, porque no se sabe qué pasa con el protagonista. Si el narradorprotagonista muriera o regresara al pasado por completo, el sentimiento compartido sería menos fuerte. La ambigüedad hace pensar en el destino del protagonista, incrementa la curiosidad y permite que el lector mantenga la empatía por más tiempo.

En segundo lugar, las fechas en el diario son un medio para mostrar la reducción de la distancia de dos conceptos opuestos. Desde el equinoccio, el protagonista con vida en el presente, entra al mundo de la mujer, de la muerte y del pasado. La distancia entre estos conceptos es más cercana que nunca al final porque el protagonista y Carlota terminan juntos en la mansión, donde se integran definitivamente el presente y el pasado. Además, como las fechas corresponden a las de las fiestas de los aztecas, se podría comparar al protagonista con un rey azteca. De ahí, deducimos que la reunión del protagonista y de la mujer sería el encuentro del mundo prehispánico y la Europa del siglo XIX, en un marco de ambigüedad donde la Emperatriz podría haberse sometido al protagonista transformado en el Rey azteca o, viceversa, si la transformación fuera hacia el carácter del Emperador.

Otros sentidos de la configuración del diario, en el caso de "Tlactocatzine, del jardín de Flandes", coinciden con un objetivo final que sería reducir las separaciones. En suma, "Tlactocatzine" es un paradigma de la literatura contemplado desde la historia por Fuentes.

\section{Referencias}

Abbott, H. P. (1982). Diary Fiction. Orbis Litterarum, 37, 12-31.

Bal, M. (1990). Teoría de la narrativa (Una introducción a la narratología). J. Franco (trad.). Madrid: Cátedra. Beltrán, L. (2011). Novela y diario. En L. P. Rodríguez Suárez, \& D. Pérez Chico (Eds.), El diario como forma de escritura y pensamiento en el mundo contemporáneo (pp. 9-19). Zaragoza: Fernando el Católico.

Brushwood, J. S. (1982). Los días enmascarados and Cantar de ciegos: Reading the Stories and Reading the Books. En R. Brody y C. Rossman (Eds.), Carlos Fuentes, a Critical View (pp. 18-33). Texas: The University of Texas.

Field, T. (1989). Form and Function in the Diary Novel. London: MacMillan.

Fuentes, C. (1970). La muerte de Artemio Cruz. (1ạ. ed. 1962). México: Fondo de Cultura Económica.

Fuentes, C. (1982). Chac Mool. En Los días enmascarados (pp. 9-27). (1a. ed. 1954). México: Era.

Fuentes, C. (1982). Tlactocatzine, del jardín de Flandes. En Los días enmascarados (pp. 34-45). (1ª. ed. 1954). México: Era. 
Fuentes, C. (2007a). Tlactocatzine, del jardín de Flandes. En Cuentos sobrenaturales (pp. 37-49). (1ä. ed. 1954). México: Alfaguara.

Fuentes, C. (2007b). Aura. En Cuentos sobrenaturales (pp. 129-180). (1ä. ed. 1962). México: Alfaguara.

García, G. (1978). Una lectura de Carlos Fuentes: Los días enmascarados; Aura. Tesis doctoral. México: El Colegio de México.

Garrido, A. (1996). El texto narrativo. Madrid: Síntesis.

Gutiérrez-Mouat, R. (1985). Carlos Fuentes y el relato fantástico. Modern Language Studies, 15 (1), 39-49.

Hall, F. (1868). Life of Maximilian I, Late Emperor of Mexico: With a Sketch of the Empress Carlota. New York: James Miller.

Ledesma, M. (2011). Principios de meteorología y climatología. Madrid: Paraninfo.

Liebowitz, B. (1986). Archetype, Architecture, and the Writer. Bloomington: Indiana University.

Kitchel, J. (n.d.). Los mayas: El equinoccio y el solsticio. Yucatán Today. Obtenido el 19 de diciembre de 2017 de: http://yucatantoday.com/los-mayas-el-equinoccio-y-el-solsticio/

López, A. (2011). Cámara de ecos: la novelística de Carlos Fuentes. Iztapalapa Revista de Ciencias Sociales y Humanidades, 71, 17-32.

Muñoz-Basols, J. (2003). La recreación del género gótico a través de la percepción sensorial: La construcción de la hipotiposis en Aura de Carlos Fuentes. Revista Atenea, 23 (2), 73-85.

Pérez, G. (1997). La configuración de elementos góticos en «Constancia», Aura y «Tlactocatzine, del jardín de Flandes» de Carlos Fuentes. Hispania, 80 (1), 9-20.

Prince, G. (1975). The Diary Novel: Notes for the Definition of a Sub-genre. Neophilologus, 59 (4), 477-481.

Quintana, M. E. (2008). Crónicas del bufón: aproximación crítica a Maluco, la novela de los descubridores. Montevideo: Linardi y Risso.

Rojas, N. (1978). Time and Tense in Carlos Fuentes' "Aura". Hispania, 61 (4), 859-864.

Sahagún, B. de. (2000). Historia general de las cosas de Nueva España, t. 1. Austin, A. López \& J. García Quintana J. (Eds.). México: Consejo Nacional para la Cultura y las Artes.

Simonson, P. (2009). Poliglotismo cultural en dos obras de Carlos Fuentes: el espacio como lenguaje. Enunciación, 14 (1), 22-34.

Tello, V. (2008). El personaje Artemio Cruz: un narrador frente al espejo de su novela. Anuario de Letras Hispánicas, 1, 89-97.

Tomasini, M. C. (2007). Astronomía, geometría y orden: el simbolismo cosmológico en la arquitectura precolombina. Colección C \& $T, 7,81-92$. 